\title{
Sistemas biomaterial-droga para la liberación controlada de antibióticos
}

\author{
Grenier L. ${ }^{1}$, González R. ${ }^{2}$
}

\begin{abstract}
RESUMEN
En el presente trabajo se recogen las principales características de los sistemas de liberación sostenida de medicamentos soportados en diferentes biomateriales y que son utilizados fundamentalmente en el tratamiento de lesiones óseas. Sobre la base de una revisión bibliográfica actualizada en este tema, se analizan en detalle los tipos de biomateriales más utilizados, así como las ventajas y desventajas que los mismos presentan en tal aplicación.
\end{abstract}

\section{SUMMARY}

The purpose of this paper was to review the main characteristics of a drug delivery System supported on different biomaterials and particularly the systems applied on the treatmet of bone diseases. The advantages and disadvantages of this type of biomaterial utilised with this objective are analysed in detail, based on the study of up-to-date literature in this area.

Actualmente, la administración local de medicamentos ha despertado gran atención debido a que constituye un método seguro y eficiente mediante el cual se obtienen efectos quimioterapéuticos superiores.

En el -caso del uso local de antibióticos, se cuenta con varias formas de aplicación como son por ejemplo: el método de irrigación-succión encerrada (1.2); perfusión regional de un miembro (3); bombas de antibióticos implantables (4), y más recientemente sistemas de liberación usando materiales que pueden retener el medicamento.

1 Licenciada en Química, Laboratorio. de Biomateriales. Centro Nacional de Investigaciones Científicas, Apdo. 6880, La Habana, Cuba.

2 Doctor en Ciencias Químicas, Investigador. Laboratorio de Biomateriales. Centro Nacional de Investigaciones Científicas, Apdo. 6880, La Habana, Cuba. (Autor al cual debe dirigirse la correspondencia).
Los sistemas de liberación controlada de medicamentos fueron concebidos debido a la necesidad de una terapia local y actualmente van adquiriendo cada vez más importancia debido a las diversas ventajas que presentan. Entre tales ventajas se encuentran las siguientes:

- Se logra una administración local de la droga.

- La acción de la droga es más efectiva, ya que a diferencia de la administración por vía oral o por perfusión, los virus u organismos extraños son atacados directamente.

- Evita segundas intervenciones.

- Se logra un mayor control de la enfermedad en cuestión.

- Los efectos secundarios perjudiciales son menores.

- Evita terapias por períodos prolongados.

- Evita administraciones simples de altas concentraciones del medicamento por vía sistémica. 
El empleo de biomateriales como soporte de medicamentos en los sistemas de liberación sostenida de la droga apropiada (5), constituye una importante aplicación que en las últimas décadas se ha venido experimentando. Esto es una consecuencia de los éxitos que se han alcanzado en el empleo de los mismos en la cirugía reconstructiva en varias especialidades, tales como maxilofacial, ortopedia, ortodoncia, etc., donde se desea la reconstrucción o reposición del tejido vivo, así como el recubrimiento de prótesis metálicas y de otros materiales.

En el presente trabajo se realiza una revisión sobre las principales combinaciones biomaterialmedicamento que se encuentran en fase de investigación y aplicación clínica, como sistemas de liberación de fármacos para el tratamiento de diferentes patologías. $Y$, de manera particular, los distintos tipos de antibióticos soportados sobre biomateriales, destinados a la terapéutica en lesiones del tejido óseo.

\section{Diferentes tipos de sistemas (biomateria-droga)}

Los biomateriales pueden ser clasificados tomando en consideración distintos aspectos. Atendiendo a su forma física y según la magnitud de la porosidad pueden ser clasificados en densos y porosos; teniendo en cuenta el tipo de reacción provocada en la interface tejido vivo-implante (6) en biotolerables, bioinertes y bioactivos; y en cuanto a su degradación en reabsorbibles y no reabsorbibles. Otra importante clasificación se relaciona con la naturaleza y composición de dichos materiales, pudiéndose citar: los metales, vidrios biológicos, polímeros sintéticos y naturales, cerámicas y otros (7).

Para la cirugía reconstructiva de las lesiones óseas, debido al avance de las investigaciones, hoy día se cuenta con materiales que presentan una morfología y composición química similares a la del hueso, lográndose éxitos en la sustitución del tejido óseo y encontrándose una alternativa que permite eliminar los inconvenientes y riesgos quirúrgicos en la práctica clínica con los diferentes tipos de injertos óseos (8).

Son varios los trabajos reportados acerca de la liberación de diversos medicamentos como los anticancerígenos (9-18) y antibióticos (19-22) incluyendo el estudio de los biomateriales en los cuales están soportadas dichas drogas.

Para estos fines, se han ensayado diferentes tipos de polímeros, composiciones de polímeros, cerámicas y biomateriales compuestos como soportes para la liberación controlada de medicamentos. En la tabla 1 se recogen, a manera de ejemplo, los principales sistemas investigados en las últimas décadas, algunos de los cuales han tenido aplicación práctica e incluso están disponibles en el mercado. Sin embargo, se está aún muy lejos de haber logrado satisfacer las necesidades de la medicina moderna en este aspecto. Es obvio que éste es un campo de investigación que recién ha comenzado y su desarrollo futuro estará en concordancia con el surgimiento y perfeccionamiento de nuevos biomateriales y fármacos.

Un tema de gran interés y preocupación mundial es el tratamiento de las enfermedades infecciosas, las lesiones sépticas y otras que requieren la aplicación de antibióticos, ya que resulta indispensable el tratamiento eficiente de estas afecciones y al mismo tiempo frenar el uso indiscriminado de este tipo de fármaco en interés de la preservación de la biosfera. Una de las formas de hacer un uso racional y efectivo de los antibióticos es precisamente su administración local y controlada y ésto se logra, cuando están soportados sobre diferentes biomateriales, en dependencia del sitio, características de la infección, objetivos del tratamiento, etc. (figura 1).

En particular, para las lesiones en el hueso, estos sistemas resultan muy efectivos, ya que como es conocido, las sepsis en este tipo de tejido son frecuentes y difíciles de tratar, requiriendo de tiempo, gran consumo de antibióticos, etc. Además, en la mayoría de los casos es necesario curar la infección antes de aplicar los procederes quirúrgicos de restauración o reconstrucción tisular lo cual se logra con estos sistemas biomaterialantibiótico de forma simultánea.

A continuación se discuten los principales sistemas biomaterial-antibiótico investigados y reportados en la literatura. 
Tabla 1. Principales biomateriales empleados en la liberación controlada de medicamentos

\begin{tabular}{|c|c|c|c|c|}
\hline Biomaterial & Medicamentos & $\begin{array}{c}\text { Exp. clínica } \\
\text { animal/humano }\end{array}$ & Año & Referencia \\
\hline \multirow{3}{*}{$\begin{array}{l}\text { Sulfato de calcio } \\
\text { (Plaster of París) }\end{array}$} & Penicilina y sulfonamida. & Humano & 1953 & $(25)$ \\
\hline & Cefazolina y lincomicina & Animal & 1992 & $(26)$ \\
\hline & Gentamicina & Animal & 1978 & $(28)$ \\
\hline \multirow[t]{4}{*}{$\begin{array}{l}\text { Polimetil-metacrilato } \\
\text { (PMMA) }\end{array}$} & Gentamicina & Humano & $\begin{array}{l}1978 \\
1981 \\
1983 \\
1985 \\
1986 \\
1990 \\
1992 \\
\end{array}$ & $\begin{array}{l}(44) \\
(19) \\
(20) \\
(31) \\
(30,37) \\
(32) \\
(21) \\
\end{array}$ \\
\hline & Tobramicina & Humano & $\begin{array}{l}1985 \\
1986 \\
1988 \\
\end{array}$ & $\begin{array}{l}(47) \\
(35) \\
(36) \\
\end{array}$ \\
\hline & Vancomicina & Humano & 1992 & (21) \\
\hline & Cefalosporina & Humano & 1992 & $(21)$ \\
\hline \multirow{3}{*}{ Acido D, L-láctico } & Didioxi-kanamicina-B & Animal & 1991 & (33) \\
\hline & Cis-platino & Animal & 1992 & $(16)$ \\
\hline & Adriamicina & Animal & $\begin{array}{l}1988 \\
1991 \\
\end{array}$ & $\begin{array}{l}(17) \\
(18) \\
\end{array}$ \\
\hline \multirow{2}{*}{ Hidroxiapatita de calcio } & Cis-platino & Animal & 1992 & $(11,12)$ \\
\hline & Gentamicina & Animal & 1992 & (62) \\
\hline $\begin{array}{l}\text { (Poli-N-acetil D-glucosamina } \\
\text { quitina) }\end{array}$ & Cis-platino & Animal & 1991 & (9) \\
\hline
\end{tabular}

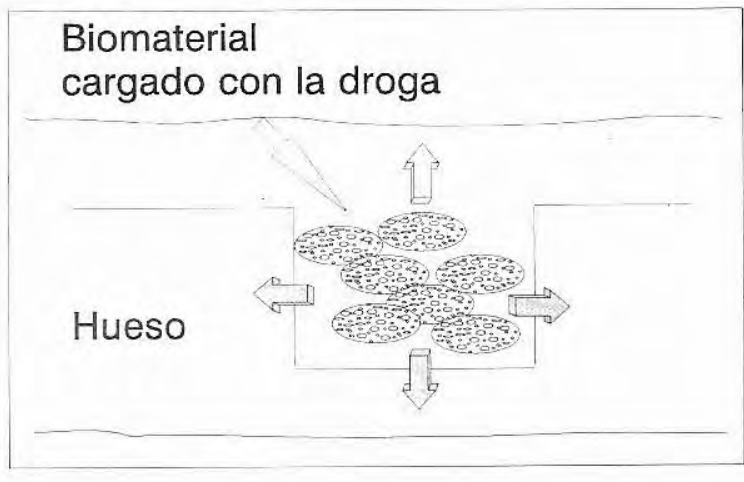

figura 1. Representación esquemática del relleno de una cavidad ósea con un biomaterial conteniendo antibiótico. El medicamento es liberado de forma controlada en todas direcciones. 


\section{Antibióticos soportados en biomateriales}

\section{El yeso (Plaster de París)}

Uno de los primeros materiales investigados como sustituto para el injerto óseo fue el yeso (CaSO4.O,5 H2O) y su producción se realiza mediante la calcinación de la gipsita natural a temperaturas de $110-130^{\circ} \mathrm{C}$ (23) con pérdida del $75 \%$ del agua.

La historia del uso del yeso como material de implante óseo ha sido revisada por varios investigadores (24-26) y aún hoy se continúa investigando y aplicando, tanto en forma directa como también formando parte de composiciones cerámicas y de cementos óseos. Las investigaciones sugieren que el yeso ocupa el espacio dejado por la lesión y evita la formación de tejidos de granulación en el sitio implantado (27). El proceso de reabsorción provoca un aumento controlado de la porosidad a la vez que se forma nuevo hueso.

El yeso fue también uno de los primeros materiales utilizados como soporte en estos sistemas, por ser un buen vehículo para la liberación de antibióticos, siendo ésto atribuido principalmente a su elevada biocompatibilidad y a su capacidad de reabsorberse. Entre los principales trabajos en este sentido se pueden mencionar los de Kovacevic, que en 1953 implantó yeso con polvo de penicilina y sulfonamida en tres pacientes (25). Posteriormente, se desarrollaron nuevas investigaciones del yeso conteniendo antibióticos $(26,28)$ concluyéndose que el yeso con antibiótico puede constituir una técnica simple para el tratamiento de infecciones óseas.

Sin embargo, a pesar de las ventajas que posee este material como la biocompatibilidad, la disponibilidad, la facilidad de uso y la economía, presenta un número de desventajas que lo hacen inferiores a otros biomateriales. Entre tales desventajas se pueden citar:

- No presenta propiedades osteoinductoras ni osteoconductoras.

- No posee mucho soporte estructural y en algunos casos tiende a perder sus propiedades mecánicas.
- Requiere de un adecuado procesamiento con una rigurosa caracterización química antes de ser usado, para eliminar las impurezas que éste presenta debido a que proviene de un mineral natural.

\section{Materiales poliméricos}

El empleo de los biomateriales poliméricos con estos fines, fue reportado por primera vez en 1970 (29) con la incorporación de antibióticos en cementos de hueso para profilaxis de infecciones postoperatorias en cirugía ortopédica. Posteriormente este sistema también ha sido utilizado para el tratamiento de osteomielitis crónica y otras infecciones en el hueso y los tejidos blandos (30-33).

En los años siguientes, varios polímeros no reabsorbibles, como el polimetilmetacrilato (PMMA) $(21,32,34,40)$, y reabsorbibles como el ácido D, L-láctico (33) fueron utilizados como soportes en la liberación de antibióticos.

Los antibióticos impregnados en cemento de hueso de polimetilmetacrilato han ganado popularidad, aunque son pocos los antibióticos que han sido adecuadamente estudiados para su uso en estos cementos (35). Sin embargo, es conocido que la adición del antibiótico en polvo al cemento de PMMA no afecta grandemente la resistencia a la tensión del cemento $(34,38)$, pero los antibióticos deben ser estables a la temperatura del cuerpo y resistentes a la degradación térmica (hasta $100^{\circ} \mathrm{C}$ ) producida durante la reacción de polimerización que ocurre cuando el cemento es mezclado.

Además, han sido estudiados en detalle los sistemas con biomateriales poliméricos como el cemento de hueso acrílico (41-48) y cemento de hueso de polipropilen-fumarato-metilmetacrilato (PPF-MMA) (49). Se reportaron algunas desventajas de estos biomateriales entre los que se incluyen:

- Reducida biocompatibilidad con el hueso.

- Corta duración de liberación.

- Muy baja proporción de liberación.

- Deterioro térmico de los antibióticos. 


\section{Materiales cerámicos (hidroxiapatita)}

Recientemente ha adquirido preponderancia el empleo de las cerámicas, debido a las ventajas que presentan las mismas con respecto a los biomateriales poliméricos.

La cerámica de hidroxiapatita de calcio porosa (HAP) ha sido difundida como material de implante en la cirugía de reconstrucción y/o sustitución ósea, mostrándose resultados satisfactorios en su aplicación.

La hidroxiapatita de calcio es el principal componente mineral del hueso, con una composición nominal de $\mathrm{Ca} 10(\mathrm{PO} 4) 6(\mathrm{OH}) 2$ y una relación molar $\mathrm{Ca} / \mathrm{P}$ de 1,67 . Se ha encontrado que las diversas formas sintéticas de hidroxiapatita resultan ser química y cristalográficamente similares a la hidroxiapatita natural del hueso (6).

Como resultado de numerosas investigaciones se ha demostrado que la hidroxiapatita de calcio presenta una excelente biocompatibilidad (5055). La biocompatibilidad de la hidroxiapatita sintética se debe no solamente a su composición, sino también por los resultados obtenidos en su implantación in vivo, donde se ha demostrado la ausencia de toxicidad, sin producir inflamación ni dolor, sangrado excesivo ni respuestas a cuerpo extraño $(56,57)$.

Se ha encontrado que la hidroxiapatita, al igual que todas las cerámicas de fosfato de calcio, son biodegradables en diferentes proporciones $(58,59)$, siendo la velocidad de biodegradación una función de la microporosidad de la cerámica (58). Por otra parte, se ha comprobado que ésta aparentemente no presenta reabsorción significativa (6).

La hidroxiapatita porosa presenta una considerable resistencia (60). Sin embargo, a pesar de que sus propiedades mecánicas son similares a la del hueso esponjoso, resulta aún muy frágil para su uso como prótesis del tejido duro. No obstante, al facilitar el crecimiento de la formación ósea hacia el inferior del implante a través de los poros, la resistencia aumenta considerablemente, lo cual posibilita que la hidroxiapatita porosa sea efectiva en el relleno de defectos óseos o como suplemento de los injertos (61).
Uchida y colaboradores, en 1992, también confirmaron la capacidad de la hidroxiapatita porosa como soporte en la liberación sostenida de medicamentos, al realizar estudios en sistemas de liberación de drogas anticancerígenas (11) y, más recientemente, en sistemas de liberación controlada de antibióticos (62) para el tratamiento de osteomielitis crónica. En tales trabajos $(11,62)$, aparecen reportadas las ventajas de este tipo de biomaterial (cerámicas) con respecto a los polímeros. Entre tales ventajas se puede citar:

- Alta biocompatibilidad.

- No tiene lugar el deterioro térmico de la droga.

- Toda la droga es liberada, no quedando parte de la misma atrapada en el compósito.

- La liberación ocurre en un período más largo.

- Presentan gran estabilidad mecánica.

- La liberación puede ser controlada teniendo en cuenta el tamaño de poro de la cerámica.

Nolan y colaboradores en investigaciones muy recientes (1993), también han ensayado con hidroxiapatita de calcio en sistemas liberadores (63).

Por otro lado, la hidroxiapatita porosa coralina HAP-200 ha sido evaluada con excelentes resultados como material de implante óseo en cirugía reconstructiva, tanto en odontología como en ortopedia, neurocirugía y otras especialidades [64] y ha comenzado a ensayarse como soporte para la liberación de antibióticos en el tratamiento de lesiones sépticas del tejido óseo (figura 2). En este caso, la composición química, forma y magnitud de la porosidad, características superficiales y otras, le confieren a este biomaterial la particularidad de poder emplearse como soporte $\mathrm{o}$, alternativamente, como contenedor de la droga o medicamento.

\section{Materiales compósitos}

Otros tipos de materiales que han sido ensayados en la liberación de antibióticos, como son los compósitos de hidroxiapatita y polímeros, tratan de combinar las propiedades positivas de la cerámica con la posibilidad de moldeabilidad y degradación más rápida del polímero, como es el caso de la hidroxiapatita con el ácido poliláctico $(65,66)$. 


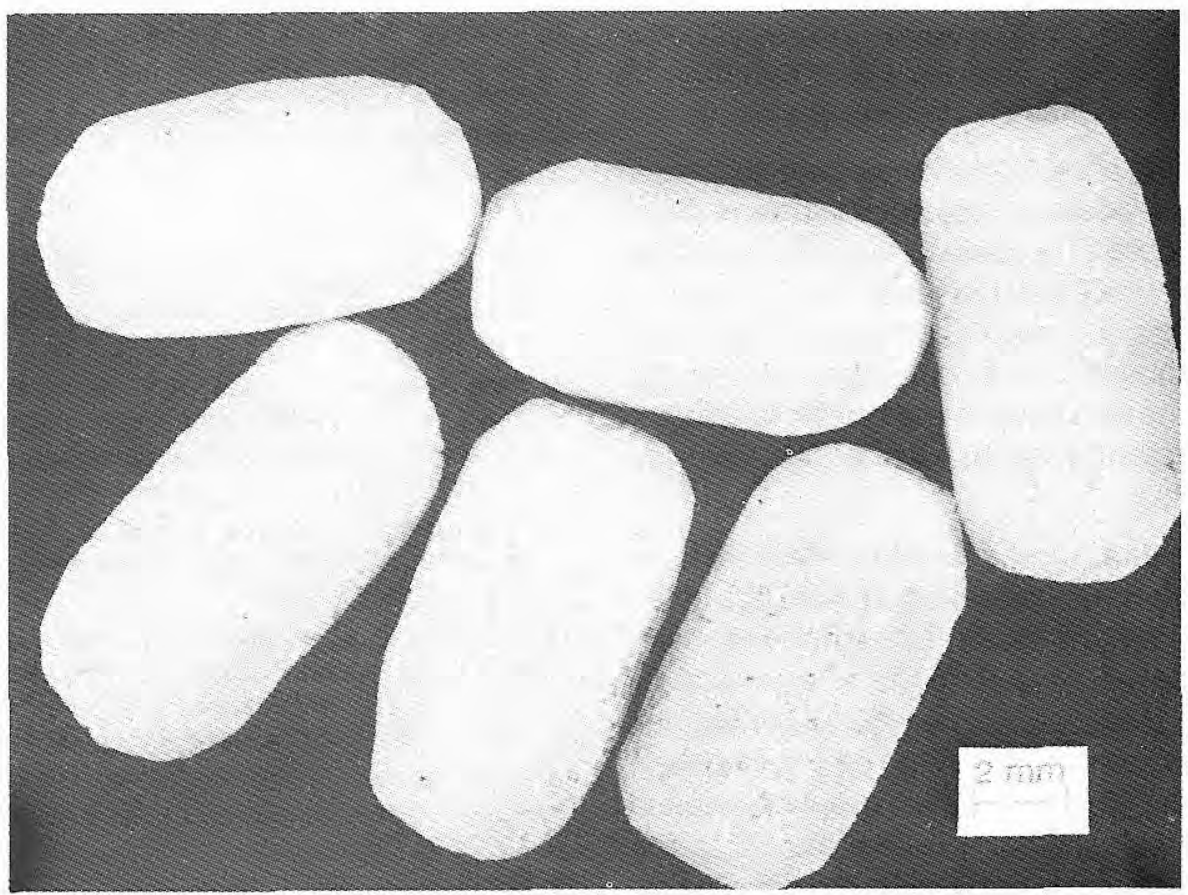

figura 2. Cápsulas de hidroxiapatita porosa coralina HAP-200 conteniendo $40 \mathrm{mg}$ de gentamicina para el tratamiento de lesiones sépticas en el hueso.

\section{Principales métodos de evaluación y aplicación de sistemas biomaterial-droga}

Estos sistemas biomaterial-droga han sido estudiados por algunos investigadores mediante experimentos in vitro e in vivo, demostrando así la efectividad y factibilidad del método.

A partir de los trabajos de Uchida $(11,12,62)$ y Wei (33), se pueden extraer algunas experiencias sobre las investigaciones tanto in vitro como preclínicas, que son necesarias para la evaluación de dichos sistemas, entre las que se encuentran:

- Los medicamentos (antibióticos o anticancerígenos) deben ser polvos o encontrarse formando compósitos con el biomaterial en cuestión.

- Los biomateriales deben tener un diámetro de microporos determinado, para que la liberación del medicamento pueda realizarse de forma sostenida y por períodos adecuados.
- Tanto en los ensayos in vitro como en modelos animales, se realizan cinéticas a través de mediciones de concentración del medicamento en el transcurso del tiempo (60-90 días).

- En los ensayos in vitro se utilizan soluciones buffer de $\mathrm{pH}$ aproximadamente 7,0 , manteniendo una temperatura similar a la del cuerpo humano $\left(37^{\circ} \mathrm{C}\right)$.

- En las experiencias preclínicas los sistemas biomaterial-droga se colocan dentro del hueso de los animales (p.e.: ratas y conejos), determinándose la concentración de la droga en el hueso, suero de sangre y otros órganos como el hígado y los riñones, haciendo uso de diferentes técnicas analíticas cuantitativas.

- Puede también ser estudiada la variación de concentración del medicamento dentro del hueso a diferentes distancias del implante.

\section{Conclusiones}

El desarrollo de biomateriales capaces de soportar y liberar de forma lenta y controlada diferentes 
fármacos, ha revolucionado la terapéutica moderna con nuevos y más eficaces métodos de tratamiento de diversas enfermedades.

De lo planteado anteriormente, se puede concluir que se conoce poco de esta aplicación y específicamente, sobre el empleo de las cerámicas. Es novedosa a nivel internacional, por lo que el curso actual de las investigaciones en este campo está encaminado a la búsqueda de nuevas cerámicas que encuentren aplicación satisfactoria en los sistemas de liberación sostenida de medicamentos.

La hidroxiapatita porosa coralina HAP-200, ha sido desarrollada recientemente y evaluada como excelente biomaterial para la reconstrucción y/o sustitución del tejido óseo $(57,67)$. Este material presenta propiedades físicas y químicas propias y particulares, conferidas por su origen y método de fabricación, por lo que presenta posibilidades potenciales de constituir un adecuado soporte para la liberación controlada de drogas y medicamentos.

\section{Referencias}

1. Grace EJ, Bryson V. Modern treatment of chronic osteomyelitis with topical detergent antibiotic therapy. Surg Gynecol Obstet 1950; 91: 333.

2. Drombrowski ET, Dunn AW. Treatment of osteomyelitis by debridement and closed wound irrigation-suction. Clin Orthop 1966; 43: 215.

3. Kelly PJ, Martin WJ, Coventry MB. Chronic osteomyelitis. II Treatment with closed irrigation and suction. JAMA 1970; 213: 1843.

4. Finsterbush $\mathbf{A}$, Weinberg $\mathbf{H}$. Venous perfusion of the limb with antibiotics for osteomyelitis and other chronic infections. J. Bone Joint Surg (Am) 1972; 1227.

5. Langer RS, Peppas NA. Present and future applications of biomaterials in controlled drug delivery systems. Biomaterials 1981; 2: 201 .

6. Heimke G. Bone replacements: Implant fixation. Argew Chem Adv Mater 1989; 1461.461: 101

7. Weber JW, White EW. Carbonate minerals as precursors of new ceramic, metal and polymer materials for biomedical applications. Min Sci Eng 1973; 5: 151.

8. González R, Guerra-López J. Materiales bioactivos para implantes óseos. Características y aplicaciones. La Habana. Edit CENIC 1993.

9. Suzuki K, Nakamura T, Tachibana M, et al. Development of slow releasing anticancer drug based with absorbable biomaterial chitin. Jpn J Cancer Chemother 1991; 18 (11): 1833
10. De Flora A, Benatti M, Guida L, et al. Encapsula-tion of adriamycin in human erythrocytes. Proc Natl Acad Sci USA 1986; 7029

11. Uchida A, Shinto $\mathrm{Y}$, Ono K, et al. Slow relase of anticancer drugs from porous calcium hydroxyapatite ceramic. J Orthop Res 1992; 10: 440.

12. Uchida A, Shinto Y, Ono K, et al. Development of a slow release system of anticancer drug retained in calcium hydroxyapatite ceramics. Jpn J Cancer Chemother 1989; 16: 3231.

13. Kato T, Nemoto R, Mori $\mathrm{H}$, et al. Sustained-release properties of microencapsulated mitomycin $\mathrm{C}$ with ethylcelulo-se infused into the renal artery of dog. Cancer $1980 ; 46: 14$

14. Sugitachi A, Takasuka $\mathrm{Y}$, Kido T, et al. A sustained released anticancer device. Trans Am Soc Artif Intern Organs 1987; 33: 626.

15. Kumieda K, et al. Implantation treatment method of slow release anticancer doxorobicin containing HA (DOXHAP) complex-A Basic study of a new treatment for hepatic cancer. $\mathrm{Br}$ cancer 1993; 67: 668 .

16. Ike $\mathrm{O}$, Shimizu Y, Wada R, et al. Controlled cis-platin delivery system using poly (D, L-lactic-acid). Biomaterials 1992; 13: 230.

17. Ike $\mathrm{O}$, Watanabe $\mathrm{S}$, Nakamura $\mathbf{T}$, et al. Relase and degradation characteristcs and antitumor effect of adriamycin containing poly-L-lactic-acid microspheres. Adv Biomater 1988; 8: 257.

18. Ike $\mathrm{O}$, Shimizu $\mathrm{Y}$, Ikada $\mathrm{Y}$, et al. Biodegradation and antitumor effect of adriamycin containing poly(L-lacticacid) microspheres. Biomaterials 1991; 12: 757.

19. Josefsson G, Lindberg L, Wiklander B. Systemic antibiotics and gentamicin-containing bone cement in the prophylaxis of post-operative infection in total hip arthosplasty. Clin Orthop 1981; 159: 194-200.

20. Christiansen K, Webb FB. The incorporation of gentamicin in bone cement; clinical studies on toxicity and therapeutic efficacy. Proceedings and reports of universities, colleges, councils, associations and societies. J Bone Joint Surg [Br] 1983; 65: 784.

21. Fish Dn, Hoffman HM, Danzinger LH. Antibioticimpregnated cement use in U.S. hospitals. Am Soc Hosp Pharmac 1992; 49: 2469.

22. Calhoun JH, Mader JT. Antibiotic beads in the management of surgical infections. Am J Surg 1989; 157:443.

23. Shinner EW, Phillips RW. Gypsum product: chemistry of setting basic principles. The science of dental materials 6th. Philadelphia: W.B Saunders Co. 1967; 51.

24. Bahn SL. Plaster: a bone substitute. Oral Surg, Oral Med, Oral Path 1966; 21: 672.

25. Peltier LF. The use of plaster of Paris to fill defects in bone. Clin Orthop 1961; 21: 1.

26. Mackey D, Varlet A, Debeaumont D. Antibiotic loaded plaster of Paris pellets: an in vivo study of a possible method of load antibiotic therapy in bone infection. Clin Orthop 1992; 167: 263. 
27. Coetzee AS. Regeneration of bone in the presence of calcium sulfate. Otolaringol 1980; 106: 405.

28. Dahners LE, Funderburk CH. Gentamicin-loaded plaster of Paris as a treatment of experimental osteomyelitis in rabbits. Clin Orthop 1987; 219: 278.

29. Buchholz HW, Engelbrecht H. Ueber die Depotwirkung einiger Antibiotica bei Vermischung mit dem Kunstharz Placos. Chirug 1970; 41: 511.

30. Salvati EA, Callaghan JJ, Brause BD, et al. Reimplantation in infection: elution of gentamicin from cement and beads. Clin Orthop 1986; 207: 83.

31. Majid SA, Lindberg LT, Gunterbert B, et al. GentamicinPMMA beads in the treatment of chronic osteomyelitis. Acta Orthop Scand 1985; 56:265.

32. Grime PD, Bowerman JE, Weller PJ. Gentamicinimpregnated polymethylmethacrylate (PMMA) beads in the treatment of primary chronic osteomyelitis of the mandible. Br J Oral Maxillofac Surg 1990; 28: 367.

33. Wei G, Kotoura Y, Oka M, et al. A bioabsorbable delivery system for antibiotic treatment of osteomyelitis. J Bone Joint Surg (Br) 1991; 73-B: 246.

34. Levin PD. The effectiveness of various antibiotics in methylmethacrylate. J Bone Joint Surg (Br) 1975; 57: 234.

35. Goodell JA, Flick AB, Hebert JC, et al. Preparation and release characteristics of tobramycin-impregnatedPMMA beads. Am J Hosp Pharm 1986; 43: 1454.

36. Scott DM, Rotschafer JC, Behrens F. Use of vancomycin and tobramycin PMMA impregnated beads in the management of chronic osteomyelitis. Drug Intell Clin Pharm 1988; 22: 480.

37. Aubrey DA, Jenkis NJ, Morgan OWP, et al. The use of gentamicin-PMMA chains in abdominal surgery: a pilot study of prophylaxis against wound infection. Pharmatherapeutica 1986; 4: 536.

38. Muehleder H, Toegel K, Urbanek H, et al. GentamicinPMMA chains in colonic surgery. Reconstr Surg Traumato! 1988; 20: 105.

39. Spence R, Anderson JR, Parks TG. The use of gentamicin-PMMA chains in colorectal surgery. Br J Clin Pract 1984; 38: 252.

40. Vecsei V, Barquet A. Treatment of chronic osteomyelitis by necretomy and gentamicin-PMMA beads. Clin Orthop 1981; 159: 201.

41. Chapman MW, Hadley WK. The effect of PMMA and antibiotic combination on bacterial viability: an in vitro and preliminary in vivo study. J Bone Joint Surg (Am) 1976; 58-A: 76.

42. Rosenthal AL, Rovell JM, Girard AE. Polyacrylic bone cement containing erthromycin and colistin. I. In vitro bacteriological activity and diffusion properties of erythromycin, colistin and erythromycin/colistin combination. J Int Med Res 1976; 4: 296.

43. Marks K, Nelson C, Lautenschlager E. Antibiotic impregnated acrylic bone cement. J Bone Joint Surg (Am) 1976; 58: 358.
44. Whaling $\mathbf{H}$, Dingelden $\mathbf{E}$, Bergmann $\mathbf{R}$, et al. The release of gentamicin from PMMA beads. J Bone Joint Surg (Br) 1978, 60-B: 270.

45. Bayston R, Milner RDR. The sustained release of antimicrobial drugs from bone cement: an appraisal of laboratory investigation and their significance. J Bone Join Surg (Br) 1982; 64-B: 460.

46. Hofman A, Krause W. Antibiotic impregnated acrylic bone cement. Orthop Trans 1982; 6: 302.

47. Kirkpatrick DK, Von Fraunhofer JA, Trachtenberg LS, et al. In vitro characteristics of tobramycin-PMMA beads: comprehensive strength and leaching. Orthop 1985;8: 1130.

48. Baker AS, Greenham LW. Release of gentamicin from acrylic bone cement: elution and diffusion studies. J Bone Joint Surg (Am) 1988; 70-A: 1551.

49. Gerhart TW, Roux RD, Horowitz G, et al. Antibiotic release from an experimental biodegradabel bone cement. J Orthop Res 1988; 6; 585.

50. Bhaskar SN, Brady JM, Getter L, et al. Biodegradable ceramic implants in bone: electron and light microscopic analysis. Oral Surg Oral Med Oral Path 1971; 32: 336.

51. Klawitter JJ, Bagwell JG, Weinstein AM, et al. An evaluation of bone growth into porous high density polyehylene. J Biomed Mater Res 1976; 10: 311.

52. Uchida A, Nade SML, Mc Cartney ER, et al. The use of ceramic for bone replacement. J Bone Joint Surg (Br) 1984; 66-B: 269-75.

53. Uchida A, Nade SML, Mc Cartney E, et al. Bone ingrowth into three different porous ceramic implanted into the tibia of rats and rabbits. J Orthop Res 1985; 3 : 65.

54. Uchida A, Nade S, Mc Cartney E, et al. Growth of bone marrow cells on porous ceramic in vitro. J Biom Mater Res 1987; $21: 1$.

55. Eggli PS, Muller W, Schenk RK. Porous hydroxyapatite and tricalcium phosphate cylinders with two different pore size ranges implanted in the cancellous bone of rabbits. Clin Orthop 1988; 232: 127.

56. Jarcho J. Calcium phosphate ceramic as hard tissue prosthetics. Clin Orthop 1981; 157: 259.

57. Socarrás E. Evaluación clínica de hidroxiapatita porosa obtenida a partir de corales marinos. Tesis de Doctorado en ciencias Médicas, La Habana 1991.

58. LeGeros RZ, Parsons JR, Dawlsi G, et al. Significance of the porosity and physical chemistry of calcium phosphate ceramic: biodegradation-biorerption. Ann NY Acad Sci 1988; 523: 268-71.

59. Bucholz RW, Carlton A, Holmes R. Interporous hidroxyapatite as a bone graft subtitute in tibial plateau fractures. Clin Orthop 1989; 240: 53.

60. Lemons JE. Hydroxyapatite coatings. Clin Orthop 1988; 235: 220.

61. Uchida A, Araki N, Shinto Y, et al. the use of calcium hydroxyapatite ceramics in bone tumour surgery. J Bone Joint Surg (Br) 1990; 72-B: 298. 
62. Shinto Y, Uchida A, Korkusuz F, et al. Calcium hydroxyapatite ceramic used as a delivery system fo antibiotics. J Bone Joint Surg (Br) 1992; 74-B: 600.

63. Nolan PC. Calcium hydroxyapatite ceramic delivery system. J Bone Joint Surg 1993; 75: 334.

64. González P. Las cerámicas de fosfato de calcio en la cirugía del tejido óseo. Estado actual y perspectivas. Biomédica 1993; 12: 2.

65. Ikada Y, Hyon SH, Jamshidi K, et al. Release of antibiotic from composites of hydroxyapatite and poly (lacticacid). J Controlled Release 1985; 2: 179.
66. Higashi S, Yamamuro T, Nakamura T, et al. PolymerHAP composites for biodegradable bone fillers. Biomaterials 1986; 7: 183.

67. Socarrás E, González R. Hidroxiapatitas y su aplicación clínica como nuevos materiales implantológicos en Cuba. Conferencia Científica del CIMEQ, C. Habana, Cuba, Marzo 1991. 